\title{
A FUZZY LOGIC MODEL FOR EVALUATION OF OIL-FILM PRESSURE IN HYDRODYNAMIC BEARING
}

\author{
Ashwani Kharola \\ Govt. Of India, Ministry of Defence \\ Institute of Technology Management (ITM), Defence R\&D Organisation \\ Uttarakhand, India
}

\begin{abstract}
This study presents a new approach for evaluation of oil-film pressure in hydrodynamic bearing. Oil-film pressure is one of the critical parameter which determines the performance of hydrodynamic bearing. In this research parameters of rotational speed, bearing load and oil-feed pressure are considered as model independent variables. Modelling and simulations are done in Matlab. The research shows that as compared to other conventional techniques approximate fuzzy reasoning provides a more logical approach.
\end{abstract}

Keywords: Oil-film pressure, Oil-feed pressure, Matlab, Simulink, fuzzy logic toolbox

\section{Introduction: Journal Bearing}

Journal Bearing permits relative motion between two parts in one or more directions with a minimum of friction while preventing motion in the direction of applied load. Classification of bearings are according to the manner in which load is supported, viz., hydrodynamic, hydrostatic and hybrid [1]. Many researchers have discussed the advantages of these bearings in their research work [2-5]. This paper, presents a fuzzy based selection strategy to predict the Oil-film pressure in hydrodynamic journal bearing. Lubrication technology is one of the key parameter for understanding journal bearings design and application[6]. One of the key parameters that describe the operating conditions in hydrodynamic lubrication is Oil-film pressure [7]. It is essential to know the expected operating condition of the bearing to gain high performance and avoid failure at crucial stages. The most commonly techniques used for bearing analysis can be categorized into rigorous and rapid techniques [8-10]. Numerical methods involves very detailed analysis of bearing geometry but tend to be expensive [11,12]. The classic one-factor-at-a-time experimental approach had been applied to evaluate journal bearing behaviour by changing one variable while the other factors are constant $[13,14]$. This paper has been divided into five parts, Part I Gives the General Introduction about the hydrodynamic bearing. Part II Explains the fuzzy logic approach for determining the Oil-Film pressure. Part III shows the Modelling and Simulation results. Part IV and Part V Conclusion and results respectively.

\section{Fuzzy Logic approach for determining Oil-Pressure in hydrodynamic journal bearing}

The fuzzy logic expert system for determining the Oil-film pressure in hydrodynamic journal bearing, the following three inputs were considered i.e. Journal bearing rotational speed ( $\mathrm{S} \mathrm{rpm}$ ),

DOI : 10.14810/ijmech.2014.3308 
Bearing load ( $\mathrm{L} \mathrm{KN}$ ) and Oil-feed pressure (P MPa), while the output parameter is Oil-film pressure (P MPa).

\subsection{Designining of the fuzzy logic expert system}

The fuzzy logic model for determination of Oil-Pressure is hydrodynamic journal bearing is shown in figure 1.0

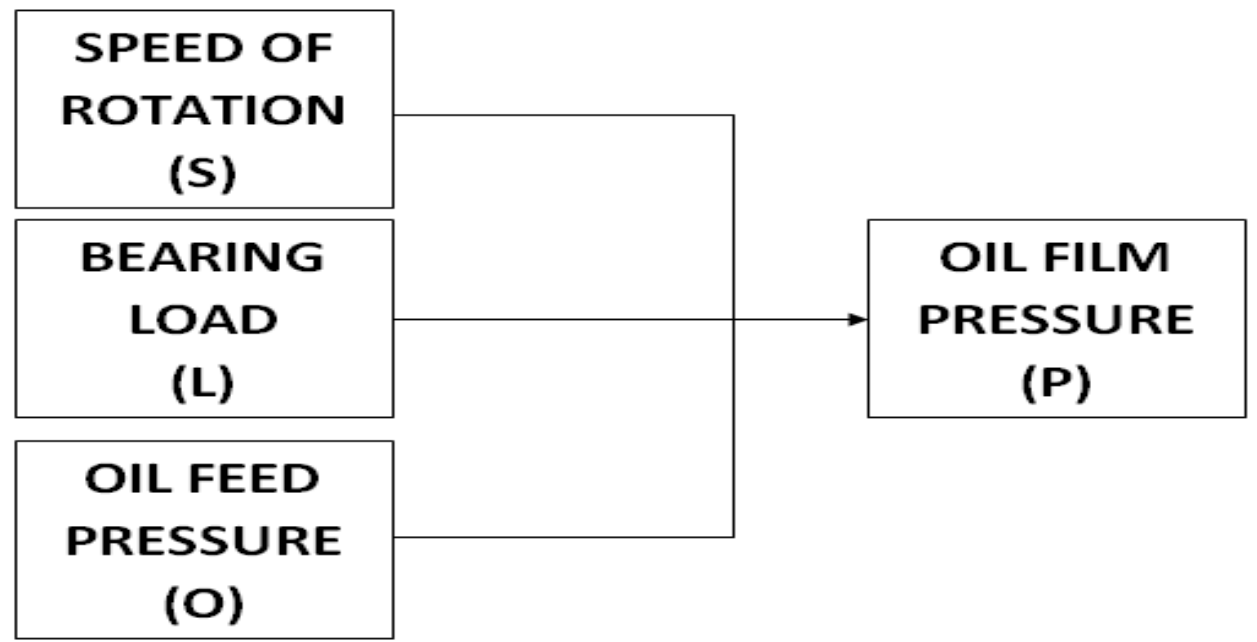

Figure 1.0 Representation of the fuzzy logic model for determination of Oil-Pressure

Mamdani inference system have been used for the modelling of the fuzzy logic controller. Figure 1.1 shows the Fuzzy Inference System (FIS) for fuzzy logic model.

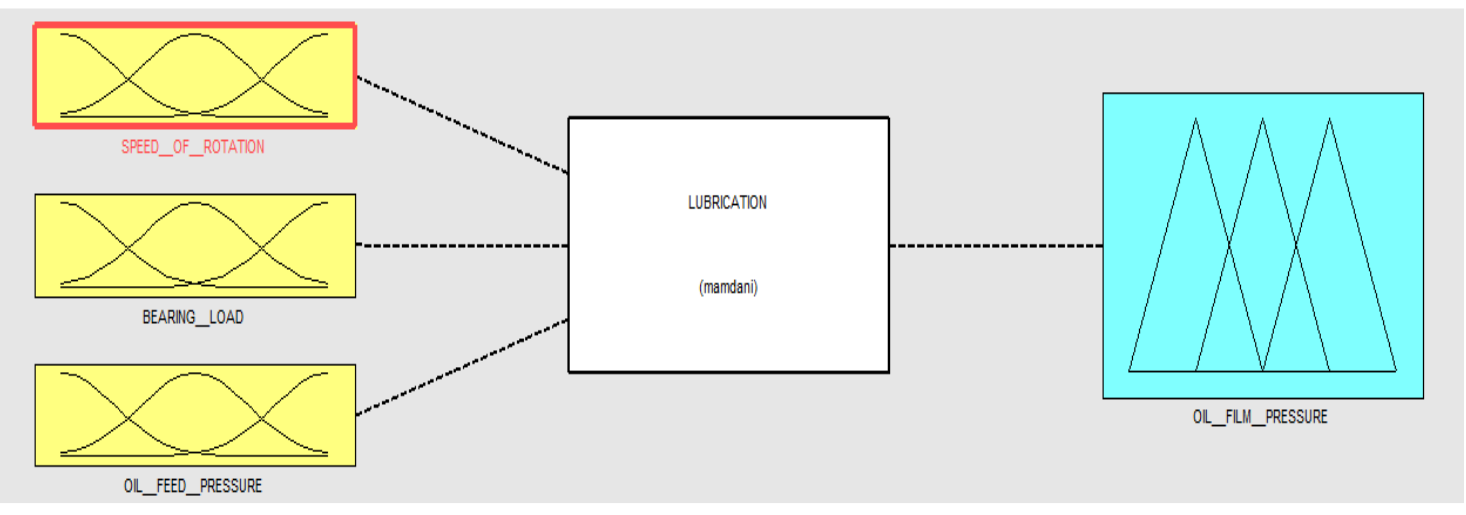

Figure 1.1 Fuzzy Inference System (FIS) for fuzzy logic model

\subsection{Defining of Fuzzy linguistic variables for the fuzzy controller}

The Fuzzy linguistic variables for the Input's/output constraint i.e. Speed of rotation (R), Bearing load (L), Oil-feed pressure $(\mathrm{O})$ and Oil-film pressure $(\mathrm{P})$ is done with five linguistic variables (fuzzy subsets: Very low-VL, Low-L, Medium-M, High-H, Very High-VH) using triangular 
MF's. Each of the constraint has been given a different UOD, examples of the fuzzified constraints are show from figure 1.2 to figure 1.6

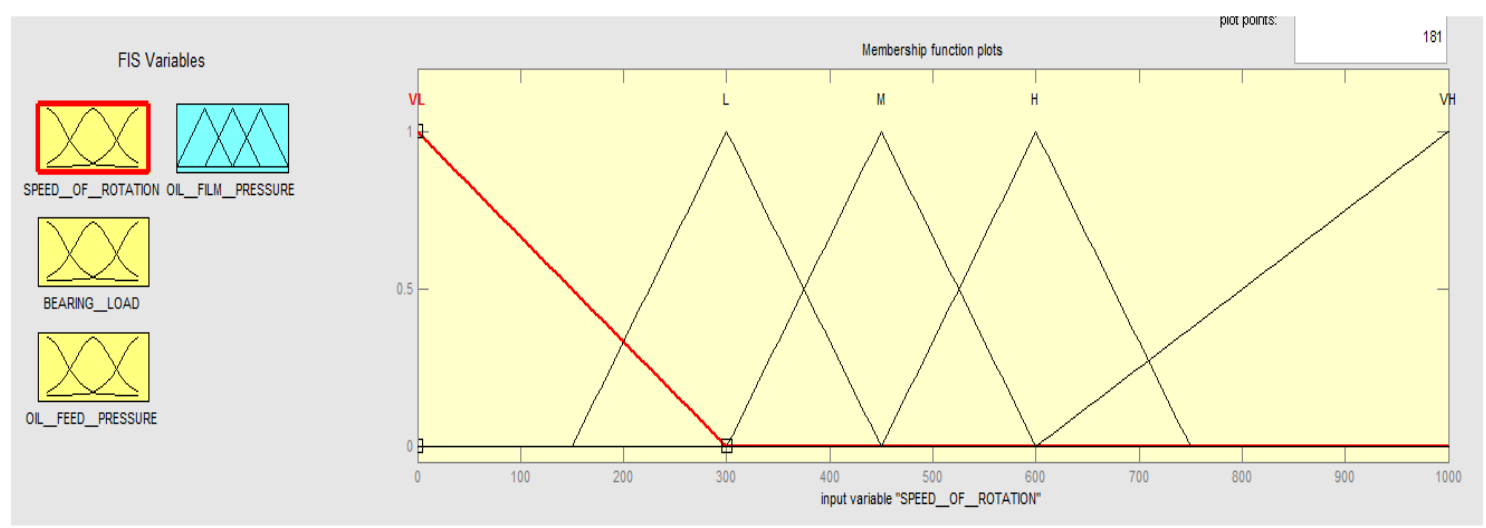

Figure 1.2 MF's for input variable ' Speed of rotation'

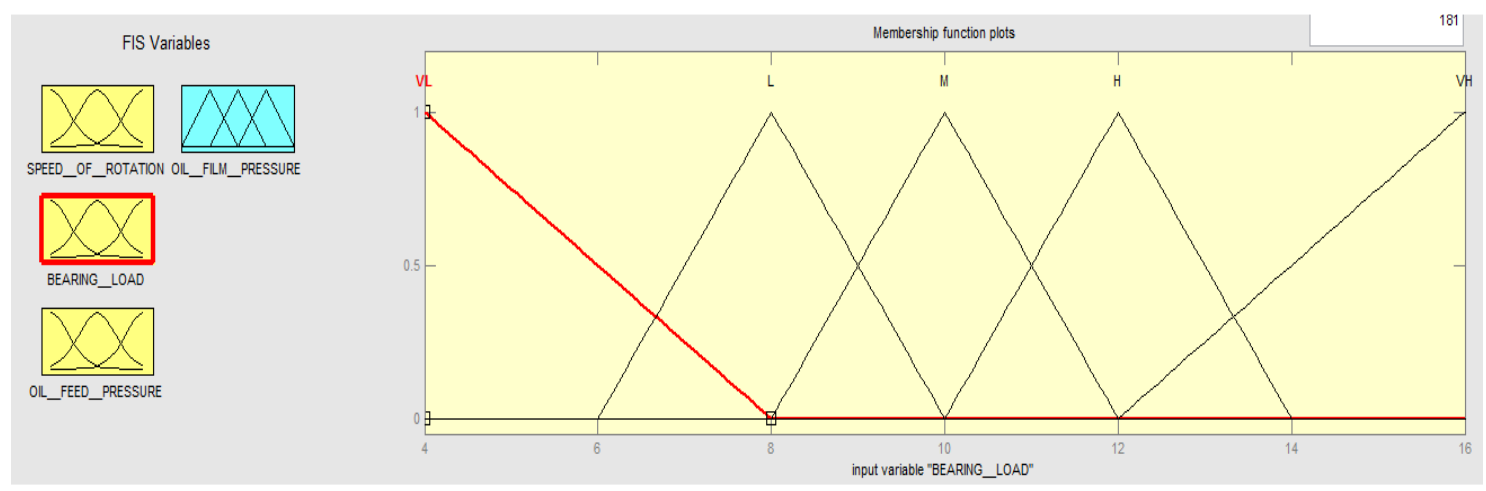

Figure 1.3 MF's for input variable ' Bearing load'

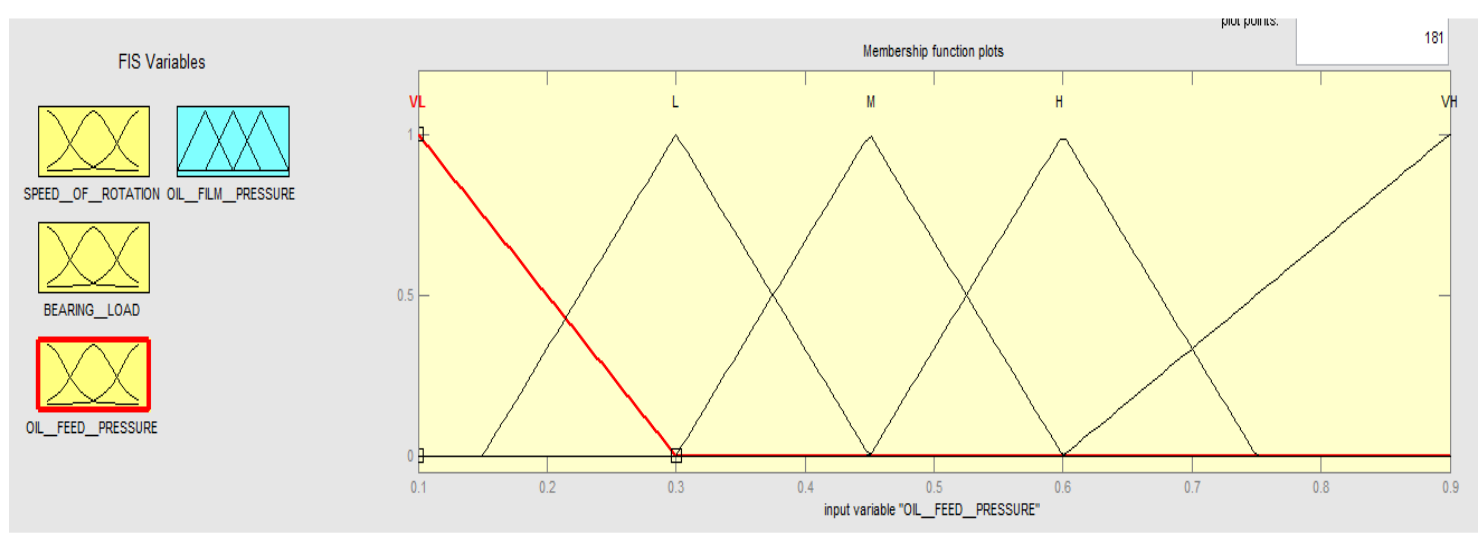

Figure 1.4 MF's for input variable ' Oil-feed pressure' 


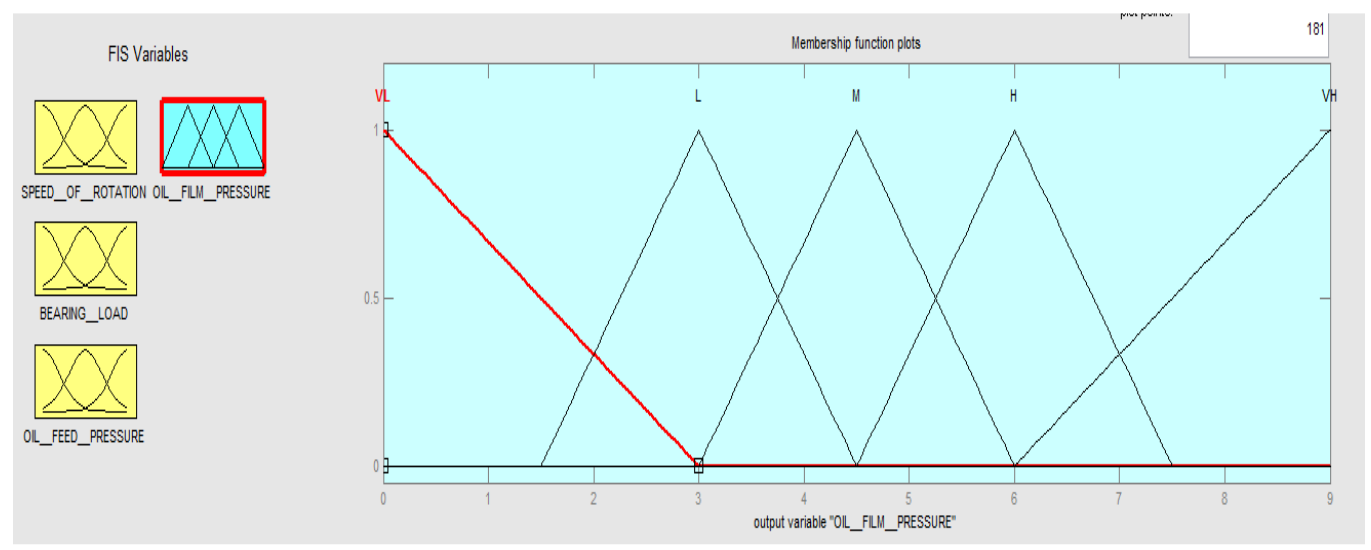

Figure 1.5 MF's for output variable 'Oil-film pressure'

\subsection{Defining fuzzy control rules}

In this study we have fuzzy ordinal approach [15] for determining fuzzy If-then rules which has reduced the number of rules to a large extent. The rules are given different degree of support (DOS) or weights which explains the relative importance of each rule. The fuzzy rules are defined and based on experience of experts. The fuzzy control rules along with DOS for the controller are given below:

(1) If 'Speed of Rotation' is 'Very low' then 'Oil film Pressure' is 'Very low' (0.5)

(2) If 'Speed of Rotation' is 'low' then 'Oil film Pressure' is 'low'(0.5)

(3) If 'Speed of Rotation' is 'Medium' then 'Oil film Pressure' is 'Medium'(0.5)

(4) If 'Speed of Rotation' is 'High' then 'Oil film Pressure' is 'High'(0.5)

(5) If 'Speed of Rotation' is 'Very high' then 'Oil film Pressure' is 'Very high'(0.5)

(6) If 'Bearing load' is 'Very low' then 'Oil film Pressure' is 'Very low' (1.0)

(7) If 'Bearing load' is 'low' then 'Oil film Pressure' is 'low' (1.0)

(8) If 'Bearing load' is 'Medium' then 'Oil film Pressure' is 'Medium' (1.0)

(9) If 'Bearing load' is 'High' then 'Oil film Pressure' is 'High' (1.0)

(10) If 'Bearing load' is 'Very high' then 'Oil film Pressure' is 'Very high' (1.0)

(11) If 'Oil feed pressure' is 'Very low' then 'Oil film Pressure' is 'Very low' (0.5)

(12) If 'Oil feed pressure' is 'low' then 'Oil film Pressure' is 'low' (0.5)

(13) If 'Oil feed pressure' is 'Medium' then 'Oil film Pressure' is 'Medium' (0.5)

(14) If 'Oil feed pressure' is 'High' then 'Oil film Pressure' is 'High' (0.5)

(15) If 'Oil feed pressure' is 'Very high' then 'Oil film Pressure' is 'Very high' (0.5)

As can be seen from above there are total 15 rules. As an example consider rule number 6 . In this rule if 'Bearing load' is 'Very low' then 'Oil film Pressure' is 'Very low' with DOS equal to 1 i.e. full membership grade. Figure 1.6 and figure 1.7 shows a surface viewer which demonstrates the relationship between the input and output variables. 
International Journal of Recent advances in Mechanical Engineering (IJMECH) Vol.3, No.3, August 2014

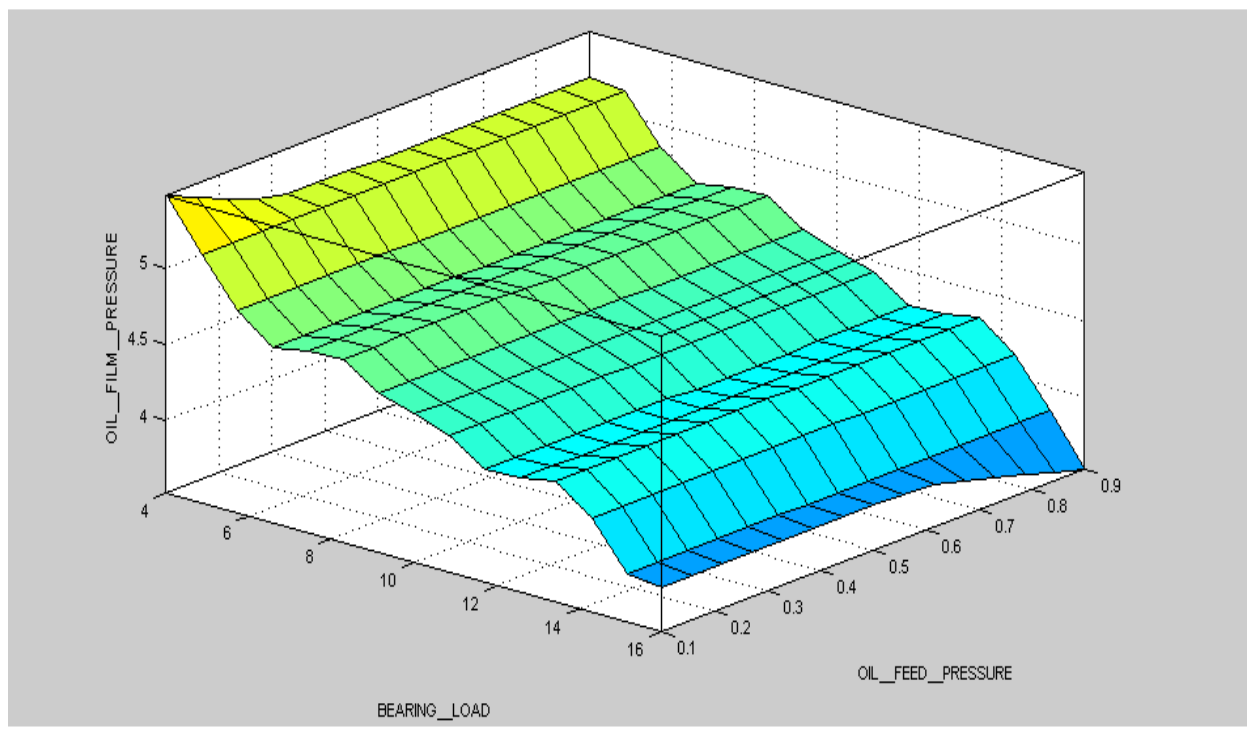

Figure 1.6 Surface viewer for Bearing load v/s Oil-feed pressure

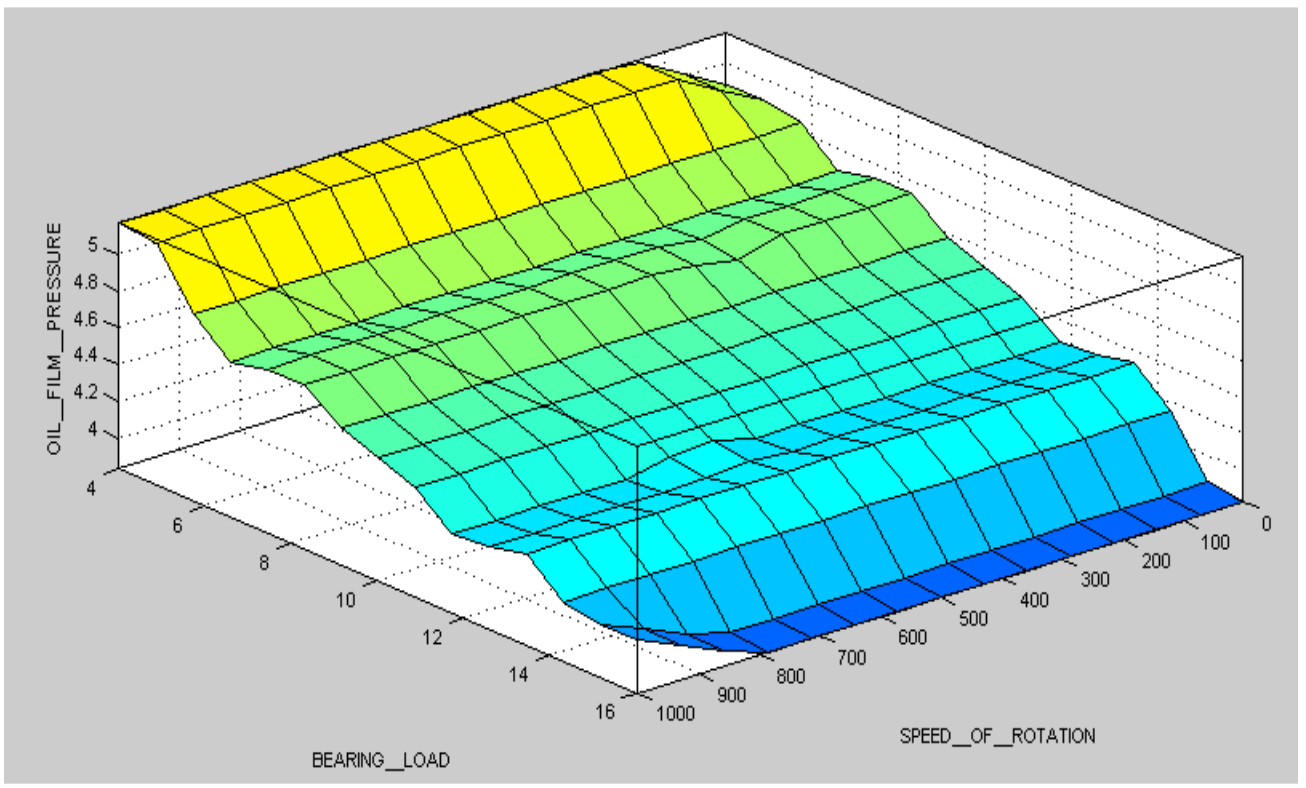

Figure 1.7 Surface viewer for Bearing load v/s Speed of rotation

\section{Modelling and Simulation results}

Simulink model for fuzzy logic controller is shown in the figure 1.8 which is built using fuzzy logic toolbox. 


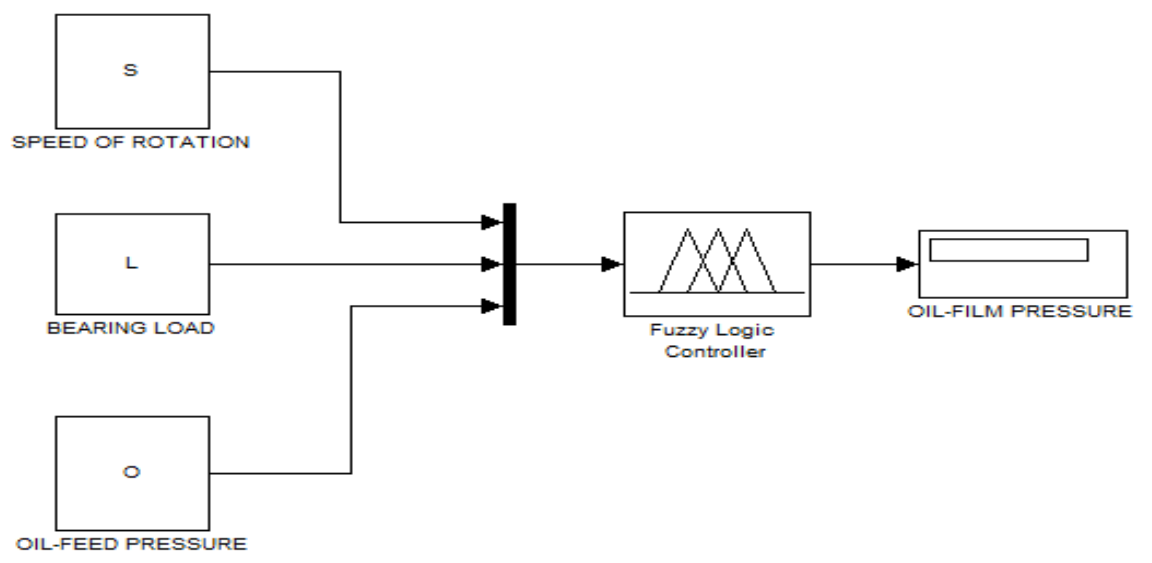

Figure 1.8 Simulink model for fuzzy logic controller

\section{Simulation results:}

\section{(1) Set 1}

\section{$S=100 ; L=10 ; O=0.75 ;$}

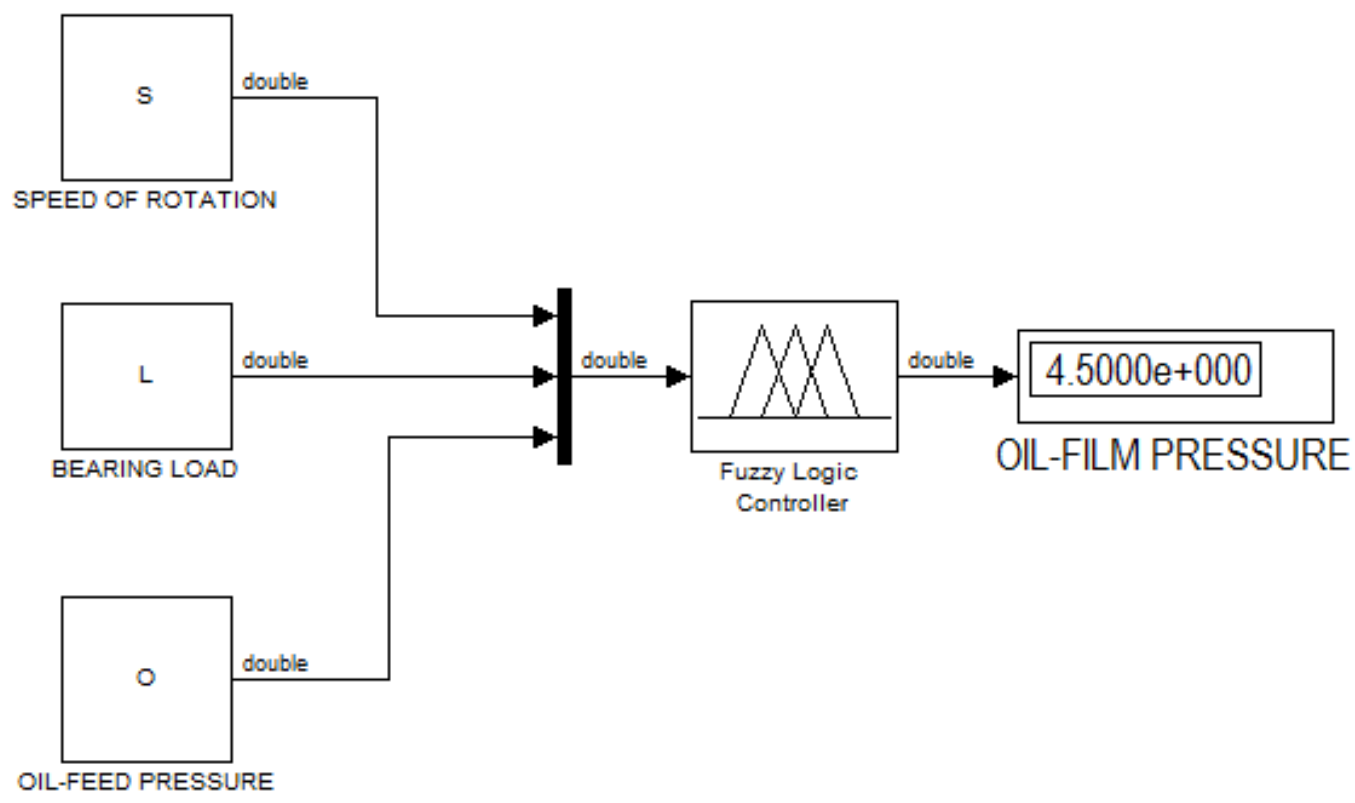

Figure 1.9 Simulation results for Set I 
(II) Set 2

$S=800 ; L=15 ; O=0.45$

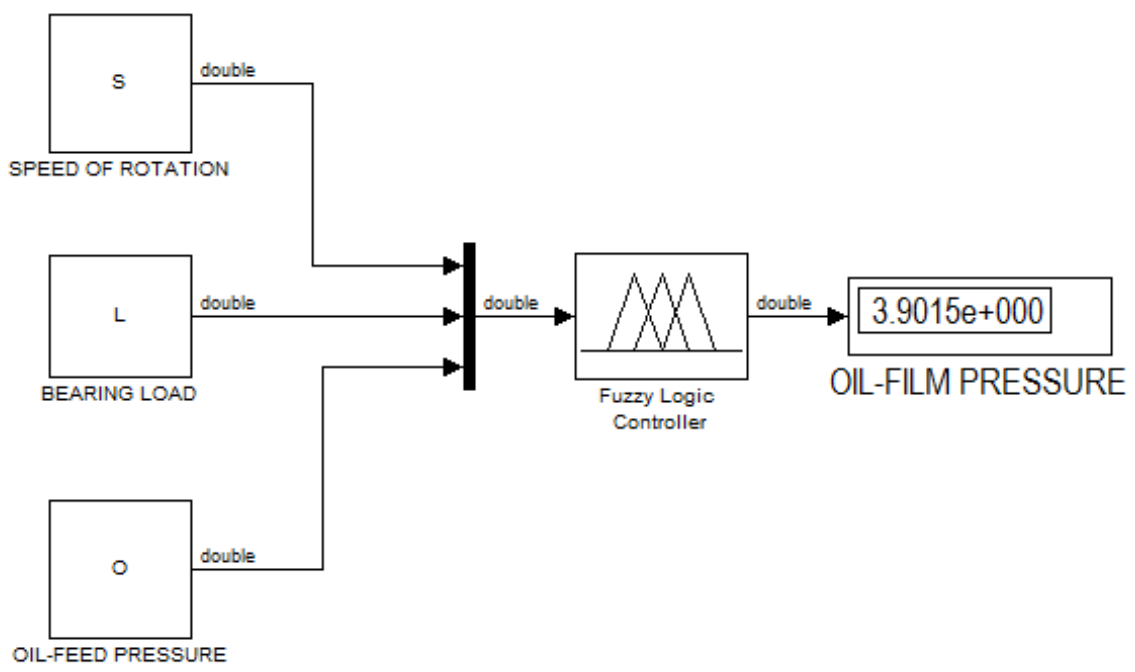

Figure 2.0 Simulation results for Set II

\section{Conclusion}

In this study a new approach for evaluation of Oil-film pressure using fuzzy logic reasoning has been proposed. Fuzzy logic reasoning provides a more logical and methodological approach to oil-film pressure evaluation as compared to other approaches. A Matlab-Simulink model has been proposed using fuzzy logic toolbox which is used for evaluation of Oil-film pressure. As an extension for future work we can also use Adaptive Neuro Fuzzy Inference System (ANFIS) based on Takagi-sugeno inference system which uses a collection of training data sets for learning and optimising the MF's and fuzzy if-then rules.

\section{References}

[1] V. K. Dwivedi, "Fuzzy based decision making for selection of fluid film journal bearing", International Journal of Fuzzy Logic Systems (IJFLS) Vol.2, No.3, July 2012

[2] Beek, A., Ostayen, R. A.J, "The design of partially grooved externally pressurized bearings", In Tribology International. Volume 39(2006) 833-838.

[3] Garg, H. C., Sharda, H.B., Kumar, "On the design and Development of Hybrid Journal Bearing", A Review. In Tribotest, Volume 12(2006) 1-19.

[4] Roy, L., Laha, S.K, "steady state and dynamic characteristics of axial grooved journal bearings", In Tribology International. Volume 42(2009) 754-761.

[5] Garg, H.C, Kumar V, Sharda H.B, "Performance of slot entry hybrid journal bearings considering combined influences of thermal effects and non- Newtonian behavior of lubricant", In Tribology International. Volume 43(2010) 1528-1531

[6] Malcolm E. Leader, " Understanding Journal Bearings", Applied Machinery Dynamics Co. Durango, Colorado

[7] Diyar I. Ahmed, S. Kasolang, Basim A. Khidhir and B.F. Yousif, " Fuzzy Logic based Model to Predict Maximum Oil-Film Pressure in Journal Bearing" , Research Journal of Applied Sciences, Engineering and Technology 6(20): 3871-3878, 2013 ISSN: 2040-7459; e-ISSN: 2040-7467 
[8] Campbell J., P.P. Love, F.A. Martin and S.O. Rafique, "Bearings for reciprocating machinery: A review of the present state of theoretical, experimental and service knowledge", Proc. Inst. Mech. Eng., 182(3A): 37-51

[9] Martin F.A., "Development in engine bearing design", Int. J. Tribology, 16: 147-164.

[10 ]Majumdar B.C "Dynamically loaded hydrodynamic fluid film bearings. Status Report on Some Selected Topics in Theoretical and Applied Mechanics", Indian National Science Academy, pp: $57-$ 64.

[11] Mahieux C, "Experimental characterization of the influence of coating materials on the hydrodynamic behaviour of thrust bearings", Int. J. Tribology, 127(3): 568-74

[12] Podevin P, G. Descombes, V. Hara, A. Clenci and C. Zaharia, "Performances of Turbochargers at Low Speeds", EAEC, Belgrade

[13] Kasolang S. and R.S. Dwyer-Joyce, "Viscosity measurement in thin lubricant films using shear ultrasonic reflection" P. I. Mech. Eng. J-J. Eng., 222(3): 423-429. , 2008

[14] Dimitrios A.B. and G.N. Pantelis, "CFD simulation of magnetorheological fluid journal bearings. Simul. Model. Pract. Th., 19(4): 1035-1060,2011.

[15] Asunobu Naitoh, Takeshi Furuhashi and Yos E Uchikawa, " A Variable Ordinal Structure Model for Fuzzy Reasoning and its Application to Decision Problem of Working Order", CH2976-9/91/0000$1539 \$ 1.0001991$ IEEE 